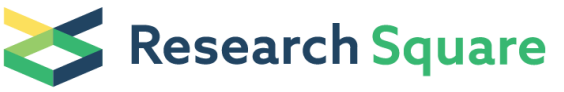 \\ Preprints are preliminary reports that have not undergone peer review. \\ They should not be considered conclusive, used to inform clinical practice, \\ or referenced by the media as validated information.
}

\section{Mutations that adapt SARS-CoV-2 to mustelid hosts do not increase fitness in the human airway.}

Jie Zhou

Imperial College London

Thomas Peacock ( $\nabla$ thomas.peacock09@imperial.ac.uk)

Department of Infectious Disease, Imperial College London https://orcid.org/0000-0001-7077-2928

Jonathan Brown

Imperial College London https://orcid.org/0000-0001-6849-3962

Daniel Goldhill

Department of Infectious Disease, Imperial College London

Ahmed Elrefaey

The Pirbright Instiute

Rebekah Penrice-Randal

Institute of Infection, Veterinary and Ecology Sciences, University of Liverpool, UK

Vanessa Cowton

University of Glasgow https://orcid.org/0000-0003-1813-7825

Giuditta De Lorenzo

MRC-University of Glasgow

Wilhelm Furnon

MRC-University of Glasgow Centre for Virus Research, Glasgow, UK

William Harvey

MRC-University of Glasgow Centre for Virus Research, Glasgow, UK

Ruthiran Kugathasan

Department of Infectious Diseases, Imperial College London, UK,

Rebecca Frise

Imperial College London

\section{Laury Baillon}

Department of Infectious Diseases, Imperial College London, UK,

Ria Lassauniere

Statens Serum https://orcid.org/0000-0002-3910-7185

Nazia Thakur

The Pirbright Institute https://orcid.org/0000-0002-4450-5911

Giulia Gallo

The Pirbright Institute, Woking, Surrey, UK, 
Institute of Infection, Veterinary and Ecology Sciences, University of Liverpool, UK

\section{l'ah Donovan-Banfield}

Institute of Infection, Veterinary and Ecology Sciences, University of Liverpool, UK

\section{Xiaofeng Dong}

Liverpool Univ

Nadine Randle

University of Liverpool

\section{Fiachra Sweeney}

Department of Infectious Disease, Imperial College London, UK,

\section{Martha Glynn}

Department of Infectious Disease, Imperial College London, UK,

\section{Jessica Quantrill}

Department of Infectious Disease, Imperial College London, UK,

\section{Paul McKay}

Imperial College London https://orcid.org/0000-0001-5195-6254

\section{Arvind Patel}

MRC-University of Glasgow Centre For Virus Research https://orcid.org/0000-0003-4600-2047

\section{Massimo Palmarini}

MRC-University of Glasgow Centre for Virus Research

\section{Julian Hiscox}

University of Liverpool

\section{Dalan Bailey}

The Pirbright Instiute

\section{Wendy Barclay}

Imperial College London https://orcid.org/0000-0002-3948-0895

\section{Article}

Keywords: SARS-CoV-2, mutations, mustelid hosts

Posted Date: August 23rd, 2021

DOI: https://doi.org/10.21203/rs.3.rs-829214/v1

License: (9) (1) This work is licensed under a Creative Commons Attribution 4.0 International License. Read Full License 


\section{Mutations that adapt SARS-CoV-2 to}

2 mustelid hosts do not increase fitness in 3 the human airway.

4 Author list: Jie Zhou ${ }^{1 \#}$, Thomas P. Peacock ${ }^{1 \#}$, Jonathan C. Brown ${ }^{1 \#}$, Daniel H. Goldhill ${ }^{1 \#}$, Ahmed M.E.

5 Elrefaey $^{2}$, Rebekah Penrice-Randal ${ }^{3}$, Vanessa M. Cowton ${ }^{4}$, Giuditta De Lorenzo ${ }^{4}$, Wilhelm Furnon ${ }^{4}$,

6 William T. Harvey ${ }^{4}$, Ruthiran Kugathasan ${ }^{1}$, Rebecca Frise ${ }^{1}$, Laury Baillon ${ }^{1}$, Ria Lassaunière ${ }^{5}$, Nazia

7 Thakur ${ }^{2,6}$, Giulia Gallo², Hannah Goldswain ${ }^{3}$, I'ah Donovan-Banfield ${ }^{3}$, Xiaofeng Dong ${ }^{3}$, Nadine P.

8 Randle $^{3}$, Fiachra Sweeney ${ }^{1}$, Martha C. Glynn ${ }^{1}$, Jessica L. Quantrill ${ }^{1}$, Paul F. McKay ${ }^{1}$, Arvind H. Patel ${ }^{4}$,

9 Massimo Palmarini ${ }^{4}$, Julian A. Hiscox ${ }^{3,7}$, Dalan Bailey ${ }^{2}$, and Wendy S. Barclay ${ }^{1}$

${ }^{1}$ Department of Infectious Disease, Imperial College London, UK.

${ }^{2}$ The Pirbright Institute, Woking, Surrey, UK.

${ }^{3}$ Institute of Infection, Veterinary and Ecology Sciences, University of Liverpool, UK.

${ }^{4} \mathrm{MRC}$-University of Glasgow Centre for Virus Research, Glasgow, UK

${ }^{5}$ Virus \& Microbiological Special Diagnostics, Statens Serum Institut, Copenhagen, Denmark.

${ }^{6}$ The Jenner Institute, Nuffield Department of Medicine, University of Oxford, UK

${ }^{7}$ Infectious Diseases Horizontal Technology Centre (ID HTC), A*STAR, Singapore.

"These authors contributed equally to this study.

*corresponding author-w.barclay@imperial.ac.uk

Keywords: SARS-CoV-2; COVID-19; coronavirus; mink; ferret; antigenicity; ACE2

\section{Abstract}

SARS-CoV-2 has a broad mammalian species tropism infecting humans, cats, dogs and farmed mink. Since the start of the 2019 pandemic several reverse zoonotic outbreaks of SARS-CoV-2 have occurred in mink, one of which reinfected humans and caused a cluster of infections in Denmark. Here we investigate the molecular basis of mink and ferret adaptation and demonstrate the spike mutations Y453F, F486L, and N501T all specifically adapt SARS-CoV-2 to use mustelid ACE2. Furthermore, we risk assess these mutations and conclude mink-adapted viruses are unlikely to pose an increased threat to humans, as Y453F attenuates the virus replication in human cells and all 3 mink-adaptations have 
minimal antigenic impact. Finally, we show that certain SARS-CoV-2 variants emerging from circulation in humans may naturally have a greater propensity to infect mustelid hosts and therefore these species should continue to be surveyed for reverse zoonotic infections.

Introduction

SARS-CoV-2 is a betacoronavirus that is thought to have emerged from an animal source in 2019 and rapidly spread by human-to-human transmission across the globe causing the COVID-19 pandemic. SARS-CoV-2 is transmitted efficiently by the airborne route due to its ability to efficiently enter cells in the upper respiratory tract. The spike glycoprotein is responsible for host receptor binding and membrane fusion of coronaviruses. SARS-CoV-2 spike binds to host angiotensinconverting enzyme 2 (ACE2) via the receptor binding domain (RBD) and is activated by TMPRSS2 protease expressed at the apical surface of the airway epithelium to mediate fusion ${ }^{1}$. In addition, compared to closely related coronaviruses, SARS-CoV- 2 spike contains a tract of basic amino acids at the $\mathrm{S} 1 / \mathrm{S} 2$ cleavage site that can be recognised by furin, enabling spike to be efficiently primed for fusion by TMPRSS2. This allows rapid fusion of spike at the cell surface and avoids restriction factors present in the late endosome and endolysosome ${ }^{2,3}$. surface of ACE2 result in SARS-CoV-2 binding to human ACE2 with high affinity ${ }^{4,5}$. SARS-CoV-2 shows a broad host tropism and can experimentally infect many animal species, largely determined by the efficiency with which spike can interact with the animal ACE2 orthologues ${ }^{5,6}$. For example, mice are inefficiently infected by early SARS-CoV-2 isolates unless they are engineered to transgenically express human ACE2 or SARS-CoV-2 is adapted to murine ACE2 by serial mouse passage 7,8 .

From April 2020, reverse-zoonotic outbreaks (i.e. transmitted from humans into animals; also known as zooanthroponosis) of SARS-CoV-2 in mink farms were reported in the Netherlands, the USA, France, Spain, Denmark, Italy, Sweden, Canada, Greece, Lithuania, and Poland ${ }^{9-13}$. Multiple reverse zoonotic events introduced the virus from farm workers into densely populated farms that then 
supported rapid transmission between animals ${ }^{12,13}$. Sequence analyses revealed several mutations in spike enriched after circulation in mink, most commonly the amino acid substitution Y453F or N501T; residues that map to the RBD of spike protein ${ }^{11-13}$. From June to November 2020 , an outbreak of SARSCoV-2 infections occurred among farmed mink in Denmark, with continuous spillover to farm workers and local communities with viruses harbouring $\mathrm{Y} 453 \mathrm{~F}{ }^{13,14}$. This large-scale outbreak resulted in an estimated 4000 mink-associated human cases and promoted the Danish government to cull all 17 million farmed mink in the country and several countries imposed total travel bans on the affected regions ${ }^{15}$. Of particular concern was the increased acquisition of spike mutations in mink-associated SARS-CoV-2 viruses, as demonstrated by the Cluster 5 variant identified in September 2020 which had several additional changes in the spike glycoprotein including, $\Delta 69-70$ in the $\mathrm{N}$-terminal domain (NTD), and $1692 \mathrm{~V}$ and $\mathrm{M} 1229 \mathrm{I}$ in S2 ${ }^{16}$. Early data indicated a possible change in antigenicity whereby Cluster 5 variant virus might be less readily neutralised by antibodies in convalescent sera from individuals infected by earlier variants ${ }^{16,17}$.

Ferrets are closely related to mink (both belong to the family Mustelidae) and have been used extensively as models for transmission of influenza virus due to their high susceptibility, comparable tissue tropism, and clinical signs similar to those seen in infected humans ${ }^{18}$. Consequently, ferrets have also been extensively characterised as models for SARS-CoV-2 transmission experiments and can support infection and transmission ${ }^{3,19,20}$. During experimental infection of SARS-CoV-2 in ferrets, several groups have independently reported mink-associated spike mutations Y453F or N501T ${ }^{12,19,21}$. Mink and ferret ACE2 are extremely similar with no amino acid differences that map to the ACE2/SARSCoV-2 Spike interface (Supplementary Figure 1). Interestingly, both the Y453F and the N501T substitutions have also been associated with increased binding to human ACE2 ${ }^{22}$. It is possible these mutations may act to non-specifically increase binding to several groups of mammalian ACE2 proteins. A similar mutation N501Y, which is also associated with increased human ACE2 binding is often found upon mouse adaptation of SARS-CoV-2 and is present in several human SARS-CoV-2 variants of concern showing signs of higher transmissibility ${ }^{23-25}$. It has been hypothesised for influenza virus that 
increasing receptor binding avidity can result in non-specific antibody escape as the viral glycoproteinhost receptor interaction begins to outcompete that of antibody/viral glycoprotein ${ }^{26}$.

In this study, we risk assess mink or ferret adapted viruses and mutations to determine the threat that viruses adapted to mustelid species could pose to humans, and what impact they could have on global vaccine efforts.

\section{Results}

Y435F and N501T substitutions in the spike are detected in viruses transmitted between ferrets.

In a previous study, we experimentally infected four donor ferrets and tested the ability of SARS-CoV-2 to transmit to individually co-housed naive animals ${ }^{3}$. The early wildtype (WT) virus isolate, England/2/2020, which contains 614D, transmitted efficiently to 2 of 4 ferrets in direct contact with two infected donor animals (donor \#1 and \#2; Figure 1A). Here, we sequenced the spike RBD of virus extracted from nasal wash obtained at early and late time point from the direct contact ferrets. Of the two transmitted virus isolates, at the consensus level, one had gained N501T in the spike protein while the other had a mixture of Y453F and N501T. Both Y453F and N501T have previously been associated with experimental ferret adaptation of SARS-CoV-2 ${ }^{19,21}$. To investigate the dynamics of ferret adaptation in more detail, virus samples were deep sequenced from various times point across the 2 successful ferret transmission chains (donor \#1 to contact \#1 and donor \#2 to contact \#2; Figure 1A). In donor animal \#1, the virus rapidly gained majority N501T with Y453F as a minor variant. However, by day 5 , both mutations were present in equal amounts with no detectable WT spike. In the matched contact animal (contact \#1), the transmitted virus population included a mixture of Y453F with a minority of N501T and Y453F continued to predominate between days 4-6. In Donor/Contact pair \#2, again both mutations were detected but N501T predominated across both animals at all time points tested. N501T alone predominated in the day 2 nasal washes from the two donor animals that did not transmit to their direct contacts ( $98 \%$ in one, $94 \%$ in the other), with remaining reads showing WT spike $^{3}$. In the initial virus inoculum, N501T was detected at levels below $1 \%$ of total reads while Y453F 
was not detected at all (read depth $\approx 7000$ ). No other consensus level mutations arose in any of the donor or contact ferrets anywhere else in the genome.

Interesting, by investigating all SARS-CoV-2 sequences isolated from mink reported on GISAID, we and others noted that N501T, Y453F, as well as F486L have independently arisen multiple times in mink, and in multiple lineages as illustrated in Figure $1 \mathrm{~B}^{11,12}$. These observations further imply that these mutations are strongly associated with mustelid adaptation (Figure 1B).

Of all the mink-adapting substitutions, Y453F has been more frequently associated with spillback from mink into humans, including Cluster 5 in Denmark. To further investigate the effects of the Y453F substitution, we isolated virus from contact \#1 from day 6 in Vero cells ('Ferret P2') and validated that the sequence change was maintained in the titrated virus stock (Figure 2A). The Vero grown virus stock was, in the majority, Y453F ( 96\%) with very minor variants, N501T and WT RBD also present $(<5 \%)$. Outside of spike, the virus contained an additional mutation, $\mathrm{S} 6 \mathrm{~L}$ in the envelope gene $(E)$ which was present in $>70 \%$ of reads in the Vero grown stock.

\section{Virus with Y453F shows enhanced replication and trended towards higher morbidity in ferrets}

To investigate whether the Y453F-containing virus showed greater replication in ferrets, we intranasally inoculated 4 naive ferrets with ferret P2 virus and compared levels of virus shed from the nose to 4 ferrets previously inoculated with the same infectious titre of parental England/2/2020 virus ${ }^{3}$ (the same donors from Figure $\left.1 \mathrm{~A}\right)$. At days 1-2, the mean titre of Y453F virus shed in nasal washes was significantly higher than that of the parental virus, as determined by both $\mathrm{E}$ gene copy number and $\mathrm{TCID}_{50}$ (Figure $2 \mathrm{~B}, \mathrm{C}$ ). Both groups of ferrets showed comparable patterns of fever during infection, peaking between days 2-4, and the Y453F-infected ferrets trended towards more weight loss over the course of the experiment (Figure 2D,E). The titre of parental virus shed and fever in parental virusinfected animals approached that in the ferret P2 infected animals by days $3-4$, likely because the parental virus had gained ferret-adapting mutations, such as Y453F or N501T, by this point (see Figure 1A). Deep sequencing of the virus from the ferrets inoculated with the Y453F-containing ferret P2 virus 

showed the Y453F substitution was maintained in all 4 animals throughout the course of infection (Figure $2 \mathrm{~F}$ ). The E gene substitution $\mathrm{S} 6 \mathrm{~L}$, however, was rapidly selected against, indicating that this substitution could have been an adaptation to cell culture, selected in Vero cells during isolation and amplification of the virus from nasal wash (Figure 2G). Several further substitutions, all present at very low levels in the inoculum, rapidly grew to fixation in all 4 Y453F-infected ferrets. These encoded mutations in spike at D614N, in N protein at R68P and in the NSP2 protein at T632I (Figure 2G). It is unclear whether these substitutions are all bona fide ferret adaptations or mutations hitchhiking as part of a selective sweep. Spike D614N may exert a similar effect to the ubiquitous SARS-CoV-2 human

\section{Y453F enhances cell entry using the mustelid ACE2 receptor}

Next, we tested whether Y453F and the other mustelid associated spike mutations improved 1692V, M1229I), all allowed SARS-CoV-2 spike expressing pseudoviruses to enter into human- or ferret, but not rat-, ACE2 expressing cells with much greater efficiency (Figure 3A, B, Extended Data Figure $2 \mathrm{~A})$. A nearby substitution, $\mathrm{L} 452 \mathrm{M}$, which has also appeared in at least one mink farm outbreaks ${ }^{11}$ has 
not dependent on the presence or absence of D614G, as Y453F in a 614D background showed a similar effect (Figure 3C, Extended data Figure 2B). Consistent results were also seen using a cell-cell fusion assay (Extended data Figure 2C,D). Examining the structure of the spike RBD/ACE2 interface, each of these mink/ferret-adaptations is close to residues that differ between human and mustelid ACE2, as others have previously modelled ${ }^{29}$. For example, Y453F lies close to H34Y (histidine in human ACE2, tyrosine in mustelid), N501T lies close to G354R, and F486L lies between ACE2 residues L79H, M82T and Q24L (Figure 3D).

Viruses containing Y453F mutation are attenuated for replication in primary human airway epithelial cells

To assess the impact of the Y453F mutation on the replication of virus in human airway epithelium, we infected primary human bronchial cells cultured at an air liquid interface with a mix of the parental and ferret P2 viruses at a low multiplicity of infection (MOI) of around 0.1 (Figure 4A). Samples were taken 24,48 and 72 hours post-infection and analysed by deep sequencing. The WT virus significantly outcompeted $\mathrm{Y} 453 \mathrm{~F}$, with less than $\sim 5 \%$ of reads by 48 hours post infection containing Y453F.

Although the Y453F containing virus is highly similar to that which circulated in mink early in the pandemic, the most prominent zoonotic spillover from mink was the Cluster 5 virus, which further contained D614G and $\Delta 69-70$. D614G and $\Delta 69-70$ are thought to potentially enhance virus infectivity in some backgrounds ${ }^{30}$. Therefore, we performed a similar competition experiment between a mixed inoculum of $40 \%$ Cluster 5 isolate and $60 \%$ early B.1 lineage, D614G containing virus ('WT'; IC19). Again, we observed that the Y453F-containing Cluster 5 was outcompeted, constituting only $~ 10 \%$ of reads by 24 hours post-infection (Figure 4B).

Finally, to further confirm that the attenuation of the Y453F containing viruses, particularly the ferret-adapted strain, wasn't due to other changes in the genome (such as E S6L described above) we generated by reverse genetics (RG) two viruses on a Wuhan-hu-1, both carrying the D614G 
mutation in spike, WT (D614G), while the other additionally contained Y453F (D614G + Y453F). As with the ferret adapted P2 virus and Cluster 5 isolate we saw that the Y453F + D614G RG virus produced less infectious virus upon replication in the primary airway cells as compared to the otherwise isogenic WT (D614G) virus, significantly so at 24 hours post-infection (Figure 4C).

\section{Mink adaptation has a minimal effect on SARS-CoV-2 antigenicity}

To investigate whether a mustelid-adapted SARS-CoV-2 crossing back into the human population would have a large impact on re-infections or vaccine-breakthrough we next tested whether the mutation at Y453F facilitated escape from antibody neutralization. Surprisingly, Y453Fcontaining 'Ferret P2' virus was significantly more easily neutralised by convalescent first wave antisera than wild type requiring only 0.6 as much antisera for a $50 \%$ neutralisation titre (Figure $5 \mathrm{~A}$ ). We further investigated the relative antigenicity of $\mathrm{Y453F}$, this time using the above-described RG viruses and antisera from health care workers who had received two doses the of Pfizer-BioNtechBNT162b2 vaccine. Again, we saw the Y453F-containing virus was more readily neutralised by 7 of the 10 vaccinee sera, although the difference was not significant (Figure 5B).

We next performed pseudovirus neutralisation assays with the previously described first wave convalescent antisera against pseudoviruses expressing the common mustelid adaptations or with full Cluster 5 spike. The B.1.351 (Beta) spike showed a significant, 5 -fold drop in mean $\mathrm{NT}_{50}$ (Figure 5C), consistent with this virus being more difficult to neutralise with first wave antisera ${ }^{31}$. None of the tested mink/ferret adaptations had any significant impact on antigenicity.

\section{Many circulating variants of concern show a greater ability to enter via mustelid ACE2.}

Following worldwide circulation of SARS-CoV-2, a number of 'variant of concern' and 'variant of interest' lineages have arisen associated with properties such as increased transmissibility, higher pathogenicity, and antigenic escape ${ }^{32}$. These generally locally, or globally, outcompeted other lineages to become predominant, including the Alpha variant (B.1.1.7), first associated with infections 
the UK ${ }^{23}$. A number of these variants have RBD mutations such as L452R, E484K and/or N501Y which are thought to promote humans ACE2 binding ${ }^{22}$.

To investigate whether these variants may be more able to infect mink or ferrets than the progenitor lineage B or B.1 viruses through better use of mustelid ACE2, we again used pseudoviruses expressing these variant spike proteins and normalised entry to human ACE2 (Figure 6). We found that nearly all variants of concern tested could better utilise mink ACE2 than WT (D614G only) pseudovirus. B.1.1.7/E484K, lota/B.1.526+E484K (first associated with infections in New York), Eta/B.1.525 (a variant with associations with West Africa) and L452R (in multiple variants of concern, including Epsilon/B.1.427/B.1.429, first associated with infections in California and Delta/B.1.617.2, which is currently replacing all other SARS-CoV-2 lineages globally) all allowed pseudovirus to utilise ferret ACE2 for cell entry to almost the same degree as human ACE2. Alpha/B.1.1.7 and Beta/B.1.351 spikes showed a much more modest boost while Gamma/P.1 (first found in Japan in travellers from Brazil) showed no improved usage of ferret ACE2. It appears L452R, E484K and N501Y may promote use of ferret ACE2, while K417N/T may result in a greater reduction in ferret ACE2 usage relative to human ACE2. Overall, these data suggest multiple circulating variants of concern may be able to infect mustelid hosts with only minimal, or indeed without, further adaptation.

\section{Discussion}

In this study, we have performed a full risk assessment of mustelid hosts, such as mink and ferrets, as reservoirs for the emergence of antigenic variants or new variant of concern. We have shown SARS-CoV-2 is poorly adapted to mustelid ACE2 and therefore quickly gains adaptations, such as Y453F, N501T or F486L to utilise mustelid ACE2. However, Y453F in particular, negatively impacts replication kinetics of SARS-CoV-2 in human cells, potentially explaining why the Danish mink-origin outbreaks did not propagate further following the culling of the mink. Furthermore, in line with other studies ${ }^{16,17,31}$, we found none of these mutations had a large antigenic impact, so vaccination is likely to remain effective against mustelid-adapted strains. Finally, we have shown that several VOC strains, 
or VOC-associated mutations, partially adapt SARS-CoV-2 spike to mustelid ACE2. Therefore, it is likely VOC lineages will continue to infect mink farms and risk spilling back over into humans.

Except for the Danish mink-adapted SARS-CoV-2 spillback, Y453F is found rarely in humans with very few isolates reported in GISAID and only a single report of the mutation arising in immunocompromised patients - this is despite Y453F having been shown in several studies to enhance human ACE2 binding, in a similar manner to the VOC-associated mutations N501Y or L452R 22,33,34. This would suggest that unlike the VOC-associated mutations such as N501Y, Y453F affects viral fitness in human cells. We have shown that, even in the presence of the putative stabilising NTD deletion, $\Delta 69-70^{30}$, virus harbouring the Y453F substitution was outcompeted by a closely related virus in human cells.

Here, we have demonstrated many VOCs, particularly Alpha/B.1.1.7 as well as those containing L452R (such as Delta/B.1.617.2) could have a fundamental fitness advantage in mink by increasing interaction with mustelid ACE2, compared to previous non-variant strains. At present (August 2021), the vast majority of mink-origin SARS-CoV-2 sequences on GISAID are from the year 2020, even though there are a number of ongoing mink outbreaks reported in Europe ${ }^{35,36}$, suggesting a significant reporting lag. None of the four WHO-designated variants of concern have yet been associated with mink farm outbreaks. It remains to be seen whether these VOCs would replicate in mink/ferrets without any further adaptation, but we have shown that the most common mustelid adaptations would be unlikely to have a large effect on VOC antigenicity. It will be key in the coming years to continue to closely survey farmed mink and to sequence and share any SARS-CoV-2 genomes from these animals in a timely manner as SARS-CoV-2 could still adapt in unexpected ways in mink ${ }^{37}$.

This work also suggests that, particularly when investigating spike RBD mutants, ferrets (or indeed mink) are poor models for humans, as mustelid ACE2 is poorly utilised by non-adapted SARSCoV-2 spike. Thus, it is not a given that adaptation to human ACE2 will also result in increased infectiousness, transmissibility or pathogenicity in the ferret model. However, ferrets remain a useful 
model for investigating non-RBD phenotypes though care should be taken to use previously ferret adapted viruses to prevent rapid adaptation.

\section{Methods}

\section{Biosafety and ethics statement}

All laboratory work was approved by the local genetic manipulation safety committee of Imperial College London, St. Mary's Campus (centre number GM77), and the Health and Safety Executive of the United Kingdom, under reference CBA1.77.20.1. SARS-CoV-2 reverse genetics work was performed at CVR University of Glasgow under HSE GM notification number is GM223/20.1a. Animal research was carried out under a United Kingdom Home Office License, P48DAD9B4. concordance with the World Medical Association's Declaration of Helsinki. Ethical approval was

Cells

African green monkey kidney cells (Vero; Nuvonis Technologies) were maintained in OptiPRO SFM (Life Tech) containing 2x GlutaMAX (Gibco). Human embryonic kidney cells (293T; ATCC; ATCC CRL-11268) were maintained in Dulbecco's modified Eagle's medium (DMEM; Gibco), 10\% fetal calf serum (FCS), 1x non-essential amino acids (NEAA; Gibco), 1x penicillin-streptomycin (P/S; Gibco). Stably transduced ACE2-expressing 293 T cells were produced as previously described ${ }^{3,39}$, and maintained with the addition of $1 \mu \mathrm{g} \mathrm{ml}-1$ puromycin to growth medium. Baby hamster kidney cells (BHK-21; ATCC CCL-10) were maintained in DMEM (Sigma-Aldrich) supplemented with 10\% FCS, 1 mM sodium pyruvate solution (Sigma-Aldrich, Germany), and 1x P/S. Air-liquid interface human airway epithelium (HAEs) cells were purchased from Epithelix and maintained in Mucilair cell culture medium 
278 (Epithelix). All cell lines were maintained at $37^{\circ} \mathrm{C}, 5 \% \mathrm{CO}_{2}$. Cell lines were not tested for mycoplasma contamination.

\section{Viruses, reverse genetics and growth kinetics}

The early SARS-CoV-2 strain, England/2/2020 (VE6-T) was previously isolated by Public Health England as previously described ${ }^{40}$. The D614G containing strain, SARS-CoV-2/England/IC19/2020, was used as previously described ${ }^{41}$. The Cluster 5 isolate - SARS-CoV-2/hu/DK/CL-5/1 - was isolated as previously described ${ }^{16}$ and was kindly provided by Kevin Bewley at Public Health England. All viral stocks used in this study were grown in Vero cells in OptiPRO SFM containing 2x GlutaMAX. Virus titration was performed by median tissue culture infectious dose $\left(\mathrm{TCID}_{50}\right)$ on Vero cells as described previously ${ }^{3}$.

Virus growth kinetics and competition assays were performed as described previously ${ }^{3,42}$.

Briefly, in air-liquid interface HAEs, before infection cells were washed with serum-free media to remove mucus and debris. Cells were infected with $200 \mu \mathrm{L}$ of virus-containing serum-free DMEM and incubated at $37^{\circ} \mathrm{C}$ for $1 \mathrm{~h}$. Inoculum was then removed and cells were washed twice. Time points were taken by adding $200 \mu \mathrm{L}$ of serum-free DMEM and incubating for 10 mins and $37^{\circ} \mathrm{C}$ before removal and titration.

Transformation-Associated Recombination (TAR) method in yeast was used to generate the mutant viruses described in this study. We followed essentially previously described methods ${ }^{43}$ with some modifications. Briefly, a set of overlapping cDNA fragments representing the entire genome of SARS-CoV-2 Wuhan isolate (GenBank: MN908947.3) were chemically synthesized and cloned into pUC57-Kan (Bio Basic Canada Inc). Where appropriate the relevant synthetic cDNA fragment carried the mutation D614G or Y453F + D614G in the viral S gene. The cDNA fragment representing the 5' terminus of the viral genome contained the bacteriophage T7 RNA polymerase promoter preceded by a short sequence stretch homologous to the Xhol-cut end of the TAR in yeast vector pEB2 ${ }^{44}$. The 
followed by a short segment homologous to the BamHI-cut end of pEB2. These cDNA fragments were excised by restriction digestion and gel-extracted or PCR-amplified using appropriate primers. These fragments were then pooled and co-transformed with Xhol-BamHI-cut pEB2 into the Saccharomyces cerevisiae strain TYC1 (MATa, ura3-52, leu2 $\Delta 1$, cyh2 $^{r}$, containing a knockout of DNA Ligase 4$)^{44}$ that had been made competent for DNA uptake using the $\mathrm{LiCl}_{2}$-based Yeast transformation kit (YEAST1$1 \mathrm{KT}$; Merck). The transformed cells were plated on minimal synthetic defined (SD) agar medium lacking uracil (Ura) but containing $0.002 \%(\mathrm{w} / \mathrm{v})$ cycloheximide to prevent selection of cells carrying the empty vector. Following incubation at $30^{\circ} \mathrm{C}$ for 4 to 5 days, colonies of the yeast transformants were screened by PCR using specific primers to identify those carrying plasmid with fully assembled genomes. Selected positive colonies were then expanded to grow in $200 \mathrm{ml} \mathrm{SD-Ura} \mathrm{dropout} \mathrm{medium}$ and the plasmid extracted as described by Thao et al. $(2020)^{43}$. Approximately $4 \mu \mathrm{g}$ of the extracted material was then used as template to in vitro synthesized viral genomic RNA transcripts using the Ribomax T7 RNA transcription Kit (Promega) and Ribo m7G Cap Analogue (Promega) as per the manufacturer's protocol. Approximately $2.5 \mu \mathrm{g}$ of the in vitro synthesized RNA was used to transfect $\sim 6 \times 10^{5}$ BHK-hACE2-N cells stably expressing the SARS-CoV-2 $\mathrm{N}$ and the human ACE2 genes ${ }^{45}$ using the MessengerMax lipofection kit (Thermo Scientific) as per the manufacturer's instructions. Cells were then incubated until signs of viral replication (syncytia formation) became visible (usually after 2-3 days), at which time the medium was collected (P0 stock) and used further as a source of rescued virus to infect VERO E6 cells to generate P1 and P2 stocks. Full genome sequences of viruses collected from from P0 and P1 stocks were obtained in order to confirm the presence of the desired mutations and exclude the presence of other spurious mutations. Viruses were sequenced using Oxford Nanopore as previously described ${ }^{46}$.

\section{E gene RT-qPCR}

Virus genomes were quantified by E gene RT-qPCR as previously described ${ }^{47}$. Viral RNA was extracted from supernatants of swab material using the QIAsymphony DSP Virus/Pathogen Mini Kit 
on the QIAsymphony instrument (Qiagen). RT-qPCR was then performed using the AgPath RT-PCR (Life Technologies) kit on a QuantStudio ${ }^{\mathrm{TM}} 7$ Flex Real-Time PCR System with the primers specific for SARSCoV-2 E gene ${ }^{48}$. For absolutely quantification of E gene RNA copies, a standard curve was generated using dilutions viral RNA of known copy number. E gene copies per ml of original virus supernatant were then calculated using this standard curve.

\section{Live virus neutralisation}

Convalescent antisera from health care workers who had tested positive by RT-qPCR were taken from the REACT2 study as described previously ${ }^{49,50}$. Double dose BNT162b2 (Pfizer-BioNtech) antisera from health care workers was generated as previously described ${ }^{38}$.

Live virus neutralisation assays were performed in Vero cells as described elsewhere ${ }^{42}$. Briefly serial dilutions of sera were incubated with $100 \mathrm{TCID}_{50}$ of virus for $1 \mathrm{~h}$ at room temperature then transferred to 96 well plates of Vero cells. Plates were incubated at $37^{\circ} \mathrm{C}, 5 \% \mathrm{CO}_{2}$ for $42 \mathrm{~h}$ before fixing cells in $4 \%$ paraformaldehyde (PFA). Cells were permeabilised in methanol $0.6 \% \mathrm{H}_{2} \mathrm{O}_{2}$ and stained for $1 \mathrm{~h}$ with an antibody against SARS-CoV-2 nucleocapsid protein (Sino Biological; 40143-R019, 1:300 dilution). Cells were further stained with the secondary antibody anti-rabbit HRP conjugate (Sigma; 1:3000 dilution) for $1 \mathrm{~h}$. TMB substrate (Europa Bioproducts) was added and developed for 20 mins before halting the reaction with $1 \mathrm{M} \mathrm{HCl}$. Plates were read at $450 \mathrm{~nm}$ and $620 \mathrm{~nm}$ and the concentration of serum needed to reduce virus signal by $50 \%$ was calculated to give NT50 values.

For the CPE-based neutralisation assay (reverse genetics virus vs Pfizer antisera), serial dilutions of sera were incubated with $100 \mathrm{TCID}_{50}$ of virus for $1 \mathrm{~h}$ at $37^{\circ} \mathrm{C}, 5 \% \mathrm{CO} 2$ in 96 well plates before a suspension of Vero-ACE2-TMPRSS2 cells were added and incubated for 3 days at $37^{\circ} \mathrm{C}, 5 \%$ $\mathrm{CO}_{2}$. Wells were stained using crystal violet, scored for the presence of virus-induced cytopathic effect and the reciprocal of the highest serum dilution at which protection was seen was calculated as the serum titre. 
Lentiviral packaging constructs pCSLW and pCAGGs-GAGPOL were made as previously

354

355

described. Mutant SARS-CoV-2 expression plasmids were generated by site-directed mutagenesis using the QuikChange Lightning Multi Site-Directed Mutagenesis Kit (Agilent). Unless otherwise stated all SARS-CoV-2 spike expression plasmids were based on the Wuhan-hu-1 reference sequence ${ }^{41}$, with the additional substitutions D614G and K1255*STOP (aka the $\Delta 19$ mutation or cytoplasmic tail truncation). Animal ACE2 proteins in pDisplay were generated and used as previously described ${ }^{5}$.

\section{Pseudovirus assays}

SARS-CoV-2 spike-bearing lentiviral pseudotypes (PV) were generated as described previously 3,28. At ICL, $100 \mathrm{~mm}$ dishes of 293Ts were transfected using lipofectamine 3000 (Thermo) with a mixture of pCSFLW, pCAGGS-GAGPOL and spike proteins expressed in pcDNA3.1. After $24 \mathrm{~h}$ supernatant was discarded and replaced. Pseudovirus-containing supernatant was collected and pooled at 48 and $72 \mathrm{~h}$ post-transfection, passed through a $0.45 \mu \mathrm{m}$ filter, aliquoted and frozen at $80^{\circ} \mathrm{C}$. At the Pirbright Institute pseudovirus was generated in 6-well plates. Cells were transfected using polyethyleneimine (PEI) with a mixture of pCSFLW, p8.91 and SARS-CoV-2 spikes expressed in pcDNA3.1. As before supernatant was discarded and replaced at $24 \mathrm{~h}$ post-transfection then harvested and pooled at 48 and $72 \mathrm{~h}$. Supernatant was clarified by low-speed centrifugation, aliquoted and frozen at $-80^{\circ} \mathrm{C}$.

Pseudovirus assays at ICL were performed as previously described ${ }^{3}$. Briefly $10 \mathrm{~mm}$ diameter dishes of 293T cells were transfected with $1 \mu \mathrm{g}$ of ACE2 of empty vector using lipofectamine 3000. 24 h later cells media was replaced, and cells were resuspended by scraping and plated into 96 well plates and overlayed with pseudovirus. $48 \mathrm{~h}$ later cells were lysed with reporter lysis buffer (Promega) and assays were read on a FLUOstar Omega plate reader (BMF Labtech) using the Luciferase Assay System (Promega). 

were transfected with $500 \mathrm{ng}$ of ACE2 or empty vector (pDISPLAY) using TransIT-X2 (Mirus Bio) according to the manufacturer's recommendation. $24 \mathrm{~h}$ later, media was removed, and cells were harvested following the addition of $2 \mathrm{mM}$ EDTA in PBS, resuspended in DMEM and plated into whitebottomed 96 wells plates (Corning). Cell were overlayed with pseudovirus $24 \mathrm{~h}$ later and incubated for $48 \mathrm{~h}$. Firefly luciferase was quantified whereby media was replaced with $50 \mu \mathrm{L}$ Bright-Glo substrate (Promega) diluted 1:2 with PBS and read on a GloMax Multi+ Detection System (Promega). CSV files were exported onto a USB flash drive for analysis.

Pseudovirus neutralisation assays were performed by incubating serial dilutions of heatinactivated human convalescent antisera with a set amount of pseudovirus. Antisera/pseudovirus mix was then incubated at $37^{\circ} \mathrm{C}$ for $1 \mathrm{~h}$ then overlayed into 96 well plates of $293 \mathrm{~T}-\mathrm{ACE} 2$ cells. Assays were then lysed and read as described above.

\section{Cell-cell fusion assay} described previously ${ }^{3}$. All ferret studies were performed in a containment level 3 laboratory, using a 
$750-1,000 \mathrm{~g}$ were used. Four donor ferrets were inoculated intranasally with $200 \mu \mathrm{l}$ of $10^{5}$ p.f.u. of each virus while lightly anaesthetized with ketamine (22 mg kg-1) and xylazine (0.9 mg kg-1).

Prior to the start of the study ferrets were confirmed to be seronegative to SARS-CoV-2. All animals were nasal-washed daily, while conscious, by instilling $2 \mathrm{ml}$ of PBS into the nostrils; the expectorate was collected into disposable $250-\mathrm{ml}$ sample pots. Ferrets were weighed daily post infection, and body temperature was measured daily via subcutaneous IPTT-300 transponder (Plexx B.V).

\section{RNA extraction and sequencing}

For Sanger sequencing, RNA was extracted from nasal washes using QIAamp viral RNA mini kit (Qiagen). RNA was reverse transcribed using Superscript IV (Invitrogen) and PCR of the spike was performed using KOD polymerase (Merck). For next generation sequencing RNA from virus-containing samples were extracted using the QIAsymphony DSP Virus/Pathogen mini kit (Qiagen). RNA was DNase-treated using the TURBO-free Kit (Invitrogen; (AM1907). cDNA was synthesised using the superscript IV reverse transcriptase (Invitrogen) and random primer mix (NEB) before amplification by the ARTIC Network protocol using the multiplexed primer scheme version 3. Fast5 files were basecalled with guppy (v.5.0.7) with high accuracy calling (hac). The fastq files produced by Nanopore sequencing were filtered with lengths 400 and 700 using Artic-ncov2019 pipeline v1.2.1 (https://artic.network/ncov-2019/ncov2019-bioinformatics-sop.htul) by "artic guppyplex" function. The other function of "artic minion" in the Artic-ncov2019 pipeline with "--medaka --medaka-model r941_min_high_g360 --normalise 0" parameters was then used to process the filtered fastq files to generate ARTIC V3 primer trimmed bam files and consensus genome sequences. These primer trimmed bam files were further analysed using DiversiTools (http://josephhughes.github.io/btctools/) with the "-orfs" function to generate the ratio of amino acid change in the reads and coverage at each site of protein in comparison to the reference SARS-CoV-2 genome (MN908947.3) as we previous description ${ }^{53}$. 
All sequences with host species labelled as Neovison vison were retrieved from the Global Initiative on Sharing All Influenza Data (GISAID) database (sequences retrieved on 7 July 2021). A table of accession IDs and acknowledgement is given in Supplementary Table S1. A sequence with only 397 nucleotides (hCoV-19/mink/Spain/NV-2105/2021, EPI_ISL_1490748) was excluded from analysis. Sequences were aligned to the Wuhan-Hu-1 reference genome sequence (MN908947) ${ }^{54}$ using MAFFT $v 7.475^{55}$ and the alignment was then checked manually. Seven further sequences were excluded from further analysis as they lacked nucleotide data enabling the determination of amino acid identity at spike positions 453, 486, 501 or 614 (hCoV-19/mink/USA/MI-CDC-II1O-7265/2020, EPI_ISL_925307; hCoV-19/mink/USA/MI-CDC-IHWB-7153/2020, EPI_ISL_925308; hCoV-19/mink/USA/WI-CDC-CX2X2436/2020, EPI_ISL_1014948; hCoV-19/mink/Netherlands/NB-EMC-3-5/2020, EPI_ISL_523094; hCoV19/mink/Netherlands/NB-EMC-3-4/2020, EPI_ISL_523093; hCoV-19/mink/Netherlands/NB-EMC-404/2020, EPI_ISL_577788; hCoV-19/mink/Denmark/mDK-56/2020, EPI_ISL_641448). Epidemiological lineages were determined using the Pangolin COVID-19 Lineage Assigner ${ }^{56,57}$ (pangolin v3.1.5, pangoLEARN v15/06/2021). Phylogenetic analysis was performed using the remaining 936 mink genomes rooted on the Wuhan-Hu-1 reference genome (MN908947) with a general time reversible model of nucleotide substitution, a proportion of invariant sites estimated from the data and a gamma distribution describing among-site rate variation (GTR $+I+\Gamma$ ) built using RAxML v8.0.0 ${ }^{58}$ with the phylogeny rooted on the sequence of the virus Wuhan-Hu-1. The maximum likelihood phylogeny was plotted, alongside data on sampling location extracted from the virus name and amino acid identity at spike positions $453,486,501$ and 614 , in R using the ggtree package ${ }^{59}$.

\section{Statistics and reproducibility} predetermine sample size. Several genome sequences were manually removed from the phylogenetic 
analysis and were described in the associated sections. The experiments were not randomized, and the investigators were not blinded to allocation during experiments and outcome assessment.

\section{Competing interests}

The authors declare no competing interests.

454

455

456

457

458

459

460

461

462

463

464

465

466

467

\section{Acknowledgements}

The SARS-CoV-2 virus isolate, England/2 was provided by Public Health England, and we thank M. Zambon, R. Gopal and M. Patel for their help. The authors would also like to thank Kevin Bewley of Public Health England for help obtaining the Cluster 5 isolate, SARS-CoV-2/hu/DK/CL-5/1 and Michelle Willicombe, Maria Prendecki and Candice Clarke for their help obtaining the Pfizer double dose antisera. We also thank E. J. Louis, University of Leicester for generously providing the TAR in yeast system. The authors thank all researchers who have shared genome data openly via the Global Initiative on Sharing All Influenza Data (GIASID).

This work was supported by the G2P-UK National Virology Consortium funded by the MRC (MR/W005611/1). Additional funding to DB, AM, NT and GG were funded by The Pirbright Institute's BBSRC institute strategic programme grant (BBS/E/I/00007038). SARS-CoV-2 research for JAH, RPR, HG, ID-B, XD and NPR is supported by the U.S. Food and Drug Administration Medical Countermeasures Initiative contract (5F40120C00085). The work at the CVR was also supported by the MRC grants (MC_UU12014/2) and the Wellcome Trust (206369/Z/17/Z).

The article reflects the views of the authors and does not represent the views or policies of the FDA.

\section{Contributions}

$J Z, T P P, J B, D H G, D B$ and WSB conceived and planned the experiments. JZ, TPP, JCB, DHG, AMEE, RP-R, VMC, GDL, WF, WTH, RK, LB, RF, RL, NT, GG, HG, ID-B, XD, NPR, FS, MCG and PFM 
TPP and WSB wrote the manuscript with input from all other authors.

\section{References}

4761 Hoffmann, M. et al. SARS-CoV-2 Cell Entry Depends on ACE2 and TMPRSS2 and Is Blocked by a Clinically Proven Protease Inhibitor. Cell 181, 271-280 e278, doi:10.1016/j.cell.2020.02.052 (2020). Hoffmann, M., Kleine-Weber, H. \& Pöhlmann, S. A Multibasic Cleavage Site in the Spike Protein of SARS-CoV-2 Is Essential for Infection of Human Lung Cells. Mol Cell 78, 779-784.e775, doi:10.1016/j.molcel.2020.04.022 (2020).

3 Peacock, T. P. et al. The furin cleavage site in the SARS-CoV-2 spike protein is required for transmission in ferrets. Nat Microbiol, doi:10.1038/s41564-021-00908-w (2021).

4 Yan, R. et al. Structural basis for the recognition of SARS-CoV-2 by full-length human ACE2. Science 367, 1444-1448, doi:10.1126/science.abb2762 (2020).

5 Conceicao, C. et al. The SARS-CoV-2 Spike protein has a broad tropism for mammalian ACE2 proteins. bioRxiv, 2020.2006.2017.156471, doi:10.1101/2020.06.17.156471 (2020).

6 Zhao, X. et al. Broad and Differential Animal Angiotensin-Converting Enzyme 2 Receptor Usage by SARS-CoV-2. Journal of Virology 94, e00940-00920, doi:10.1128/jvi.00940-20 (2020).

$7 \mathrm{Gu}, \mathrm{H}$. et al. Adaptation of SARS-CoV-2 in BALB/c mice for testing vaccine efficacy. Science 369, 1603-1607, doi:10.1126/science.abc4730 (2020).

8 Rathnasinghe, R. et al. The N501Y mutation in SARS-CoV-2 spike leads to morbidity in obese and aged mice and is neutralized by convalescent and post-vaccination human sera. medRxiv, 2021.2001.2019.21249592, doi:10.1101/2021.01.19.21249592 (2021).

9 OIE. Events in animals, <https://www.oie.int/en/scientific-expertise/specific-informationand-recommendations/questions-and-answers-on-2019novel-coronavirus/events-inanimals/> (2021).

10 Rabalski, L. et al. Detection and molecular characterisation of SARS-CoV-2 in farmed mink (<em>Neovision vision</em>) in Poland. bioRxiv, 2020.2012.2024.422670, doi:10.1101/2020.12.24.422670 (2020).

11 Lu, L. et al. Adaptation, spread and transmission of SARS-CoV-2 in farmed minks and related humans in the Netherlands. bioRxiv, 2021.2007.2013.452160, doi:10.1101/2021.07.13.452160 (2021).

12 Oude Munnink, B. B. et al. Transmission of SARS-CoV-2 on mink farms between humans and mink and back to humans. Science, doi:10.1126/science.abe5901 (2020).

13 Hammer, A. S. et al. SARS-CoV-2 Transmission between Mink (Neovison vison) and Humans, Denmark. Emerg Infect Dis 27, 547-551, doi:10.3201/eid2702.203794 (2021).

14 Larsen, H. D. et al. Preliminary report of an outbreak of SARS-CoV-2 in mink and mink farmers associated with community spread, Denmark, June to November 2020. Euro Surveill 26, doi:10.2807/1560-7917.Es.2021.26.5.210009 (2021).

15 Ministry of Environment and Food of Denmark. COVID-19: all mink in Denmark must be culled, $<$ https://en.mfvm.dk/news/news/nyhed/covid-19-all-mink-in-denmark-must-be-culled/> (2020).

16 Lassaunière, R. et al. In vitro Characterization of Fitness and Convalescent Antibody Neutralization of SARS-CoV-2 Cluster 5 Variant Emerging in Mink at Danish Farms. Frontiers in Microbiology 12, doi:10.3389/fmicb.2021.698944 (2021).

17 Hoffmann, M. et al. SARS-CoV-2 mutations acquired in mink reduce antibody-mediated 
Belser, J. A. et al. Ferrets as Models for Influenza Virus Transmission Studies and Pandemic Risk Assessments. Emerg Infect Dis 24, 965-971, doi:10.3201/eid2406.172114 (2018). Richard, M. et al. SARS-CoV-2 is transmitted via contact and via the air between ferrets. Nat Commun 11, 3496, doi:10.1038/s41467-020-17367-2 (2020).

20 Kim, Y. I. et al. Infection and Rapid Transmission of SARS-CoV-2 in Ferrets. Cell Host Microbe 27, 704-709 e702, doi:10.1016/j.chom.2020.03.023 (2020).

21 Everett, H. E. et al. Intranasal Infection of Ferrets with SARS-CoV-2 as a Model for Asymptomatic Human Infection. Viruses 13, 113 (2021).

22 Starr, T. N. et al. Deep Mutational Scanning of SARS-CoV-2 Receptor Binding Domain Reveals Constraints on Folding and ACE2 Binding. Cell 182, 1295-1310.e1220, doi:https://doi.org/10.1016/j.cell.2020.08.012 (2020).

23 Rambaut, A. et al. Preliminary genomic characterisation of an emergent SARS-CoV-2 lineage in the UK defined by a novel set of spike mutations. (virological.org, 2020).

24 Tegally, H. et al. Emergence and rapid spread of a new severe acute respiratory syndromerelated coronavirus 2 (SARS-CoV-2) lineage with multiple spike mutations in South Africa. medRxiv, 2020.2012.2021.20248640, doi:10.1101/2020.12.21.20248640 (2020).

25 Naveca, F. et al. SARS-CoV-2 reinfection by the new Variant of Concern (VOC) P.1 in Amazonas, Brazil. (virological.org, 2021).

26 Hensley, S. E. et al. Hemagglutinin receptor binding avidity drives influenza A virus antigenic drift. Science 326, 734-736, doi:10.1126/science.1178258 (2009).

27 Juraszek, J. et al. Stabilizing the closed SARS-CoV-2 spike trimer. Nature Communications 12, 244, doi:10.1038/s41467-020-20321-x (2021).

28 Conceicao, C. et al. The SARS-CoV-2 Spike protein has a broad tropism for mammalian ACE2 proteins. PLoS Biol 18, e3001016, doi:10.1371/journal.pbio.3001016 (2020).

29 Welkers, M. R. A., Han, A. X., Reusken, C. B. E. M. \& Eggink, D. Possible host-adaptation of SARS-CoV-2 due to improved ACE2 receptor binding in mink. Virus Evolution, doi:10.1093/ve/veaa094 (2020).

30 Meng, B. et al. Recurrent emergence of SARS-CoV-2 spike deletion H69/V70 and its role in the Alpha variant B.1.1.7. Cell Reports, 109292, doi:https://doi.org/10.1016/j.celrep.2021.109292 (2021).

31 Garcia-Beltran, W. F. et al. Multiple SARS-CoV-2 variants escape neutralization by vaccineinduced humoral immunity. Cell 184, 2372-2383.e2379, doi:10.1016/j.cell.2021.03.013 (2021).

32 Peacock, T. P., Penrice-Randal, R., Hiscox, J. A. \& Barclay, W. S. SARS-CoV-2 one year on: evidence for ongoing viral adaptation. J Gen Virol 102, doi:10.1099/jgv.0.001584 (2021).

33 Motozono, C. et al. SARS-CoV-2 spike L452R variant evades cellular immunity and increases infectivity. Cell Host \& Microbe 29, 1124-1136.e1111, doi:https://doi.org/10.1016/i.chom.2021.06.006 (2021).

34 Bayarri-Olmos, R. et al. The SARS-CoV-2 Y453F mink variant displays a pronounced increase in ACE-2 affinity but does not challenge antibody neutralization. Journal of Biological Chemistry 296, 100536, doi:https://doi.org/10.1016/j.jbc.2021.100536 (2021).

35 Renewed calls for closure of Galicia mink farms after four more Covid outbreaks, $<$ https://spanishnewstoday.com/renewed-calls-for-closure-of-galicia-mink-farms-after-fourmore-covid-outbreaks 1612810-a.html> (2021).

36 Poland orders cull at fur farm with country's first mink coronavirus case, $<$ https://notesfrompoland.com/2021/02/01/poland-orders-cull-at-fur-farm-with-countrysfirst-mink-coronavirus-case/> (2021).

37 Goldhill, D. H. \& Barclay, W. S. 2020 hindsight - Should evolutionary virologists have expected the unexpected during a pandemic? Evolution, doi:10.1111/evo.14317 (2021). 
38 Prendecki, M. et al. Humoral and T-cell responses to SARS-CoV-2 vaccination in patients receiving immunosuppression. Ann Rheum Dis, doi:10.1136/annrheumdis-2021-220626 (2021).

39 Rebendenne, A. et al. SARS-CoV-2 triggers an MDA-5-dependent interferon response which is unable to control replication in lung epithelial cells. Journal of Virology, JVI.02415-02420, doi:10.1128/jvi.02415-20 (2021).

40 Davidson, A. D. et al. Characterisation of the transcriptome and proteome of SARS-CoV-2 reveals a cell passage induced in-frame deletion of the furin-like cleavage site from the spike glycoprotein. Genome Med 12, 68, doi:10.1186/s13073-020-00763-0 (2020).

41 McKay, P. F. et al. Self-amplifying RNA SARS-CoV-2 lipid nanoparticle vaccine candidate induces high neutralizing antibody titers in mice. Nat Commun 11,3523, doi:10.1038/s41467020-17409-9 (2020).

42 Brown, J. C. et al. Increased transmission of SARS-CoV-2 lineage B.1.1.7 (VOC 2020212/01) is not accounted for by a replicative advantage in primary airway cells or antibody escape. bioRxiv, 2021.2002.2024.432576, doi:10.1101/2021.02.24.432576 (2021).

43 Thi Nhu Thao, T. et al. Rapid reconstruction of SARS-CoV-2 using a synthetic genomics platform. Nature 582, 561-565, doi:10.1038/s41586-020-2294-9 (2020).

44 Gaida, A. et al. Cloning of the Repertoire of Individual Plasmodium falciparum var Genes Using Transformation Associated Recombination (TAR). PLOS ONE 6, e17782, doi:10.1371/journal.pone.0017782 (2011).

45 Rihn, S. J. et al. A plasmid DNA-launched SARS-CoV-2 reverse genetics system and coronavirus toolkit for COVID-19 research. PLoS Biol 19, e3001091, doi:10.1371/journal.pbio.3001091 (2021).

46 da Silva Filipe, A. et al. Genomic epidemiology reveals multiple introductions of SARS-CoV-2 from mainland Europe into Scotland. Nature Microbiology 6, 112-122, doi:10.1038/s41564020-00838-z (2021).

47 Zhou, J. et al. Investigating SARS-CoV-2 surface and air contamination in an acute healthcare setting during the peak of the COVID-19 pandemic in London. Clin Infect Dis, doi:10.1093/cid/ciaa905 (2020).

48 Corman, V. M. et al. Detection of 2019 novel coronavirus (2019-nCoV) by real-time RT-PCR. Eurosurveillance 25, 2000045, doi:doi:https://doi.org/10.2807/15607917.ES.2020.25.3.2000045 (2020).

49 Flower, B. et al. Clinical and laboratory evaluation of SARS-CoV-2 lateral flow assays for use in a national COVID-19 seroprevalence survey. Thorax 75, 1082-1088, doi:10.1136/thoraxjnl2020-215732 (2020).

50 Moshe, M. et al. SARS-CoV-2 lateral flow assays for possible use in national covid-19 seroprevalence surveys (React 2): diagnostic accuracy study. BMJ 372, n423, doi:10.1136/bmj.n423 (2021).

51 Thakur, N. et al. Micro-fusion inhibition tests: quantifying antibody neutralization of virusmediated cell-cell fusion. Journal of General Virology 102, doi:https://doi.org/10.1099/jgv.0.001506 (2021).

52 Ishikawa, H., Meng, F., Kondo, N., Iwamoto, A. \& Matsuda, Z. Generation of a dual-functional split-reporter protein for monitoring membrane fusion using self-associating split GFP. Protein Eng Des Sel 25, 813-820, doi:10.1093/protein/gzs051 (2012).

53 Dong, X. et al. Variation around the dominant viral genome sequence contributes to viral load and outcome in patients with Ebola virus disease. Genome Biology 21, 238, doi:10.1186/s13059-020-02148-3 (2020).

54 Wu, F. et al. A new coronavirus associated with human respiratory disease in China. Nature 579, 265-269, doi:10.1038/s41586-020-2008-3 (2020). 
Katoh, K. \& Standley, D. M. MAFFT multiple sequence alignment software version 7: improvements in performance and usability. Mol Biol Evol 30, 772-780, doi:10.1093/molbev/mst010 (2013).

56 Rambaut, A. et al. A dynamic nomenclature proposal for SARS-CoV-2 lineages to assist genomic epidemiology. Nat Microbiol 5, 1403-1407, doi:10.1038/s41564-020-0770-5 (2020). O'Toole, Á. et al. Assignment of epidemiological lineages in an emerging pandemic using the pangolin tool. Virus Evolution, doi:10.1093/ve/veab064 (2021).

58 Stamatakis, A. RAxML version 8: a tool for phylogenetic analysis and post-analysis of large phylogenies. Bioinformatics 30, 1312-1313, doi:10.1093/bioinformatics/btu033 (2014).

59 Yu, G., Smith, D. K., Zhu, H., Guan, Y. \& Lam, T. T.-Y. ggtree: an r package for visualization and annotation of phylogenetic trees with their covariates and other associated data. Methods in Ecology and Evolution 8, 28-36, doi:https://doi.org/10.1111/2041-210X.12628 (2017).

631

\section{Figure legends}

Figure 1. Passage of SARS-CoV-2 in ferrets results in spontaneous emergence of the mink-associated

\section{mutations Y453F and N501T.}

A) Ferret transmission chains from a previous study were deep sequenced to investigate any changes that occurred during infection and transmission of ferrets. Grey lines indicate previously described RNA shedding patterns seen in each ferret - pie charts indicate RBD mutations seen at each time point (as determined by deep sequencing) indicated by a black arrow.

B) Maximum-likelihood phylogeny of SARS-CoV-2 genomes sampled from American mink (Neogale vison, formerly Neovison vison), highlighting the spike mutations del69-70, Y453F, F486L or F486I, N501T, and D614G. Tip nodes are shown as points coloured by sampling location, according to the colour key. Columns to the right show the presence of either the wild-type amino acid(s) (light grey) or the mutations annotated above (coloured bars). Major epidemiological lineages designated with the Pango nomenclature system are labelled. Black arrow indicates the branch that constitutes the Danish mink strain known as cluster 5 . At position 486, mutant viruses possessed $486 \mathrm{~L}$ (leucine) except for a monophyletic clade formed of 20 sequences sampled in Latvia that possessed $486 \mathrm{I}$ (isoleucine) that are marked by a white asterisk. 

1a) before and after isolation in Vero cells. (orange circles; as previously described in ${ }^{3}$ ) or Y453F (ie ferret passage 2; black and white squares) SARS-CoV-2. $\mathrm{N}=4$ naïve ferrets in each group were infected with $10^{5}$ p.f.u. of either virus. Percentage weight loss (D) and change in body temperature (E) were recorded daily. Statistics on B and C determined by multiple Mann-Whitney tests. ${ }^{*} 0.05 \geq P$ from Figure $2 A-D$ and dynamics over time in directly infected ferrets.

Figure 3. Mink- and ferret-associated spike mutations allow more efficient entry into cells expressing the ferret ACE2 receptor.

A) Pseudovirus entry in human or ferret ACE2 expressing cells. Mutant SARS-CoV-2 spikecontaining pseudovirus entry into HEK 293Ts expressing human or ferret ACE2 or empty vector. Entry normalised to signals from human ACE2 expressing cells. Each data point indicates mean value taken from a completely independent repeat $(\mathrm{N} \geq 3)$. Statistics were determined by comparing logtransformed values of ferret ACE2 entry using a one-way ANOVA with multiple comparisons against the WT. ${ }^{*} 0.05 \geq P>0.01 ;{ }^{* *} 0.01 \geq P>0.001 ;{ }^{* * *} 0.001 \geq P>0.0001 ; * * * * P \leq 0.0001$. Entry of SARS-CoV-2 spike mutant-expressing lentiviral pseudotypes into BHK-21 cells expressing different mammalian ACE2 proteins. Pseudovirus shown contain either D614G (B) or D614 $\mathrm{N} \geq 3$ repeats. 

residues that differ in mustelid and human ACE2. Figure made using PyMOL (Schrödinger) and PDB: $7 A 94^{60}$ human airway cells. Human primary airway epithelial cells cultured at air-liquid interface were infected at an MOI of approximately 0.1 with A) a mixture of parental and ferret-adapted England/2 virus B) A mixture of Mink-adapted 'Cluster 5' virus and a D614G control or C) either isogenic WT (D614G) or D614G + Y453F -containing reverse genetics-derived virus isolates. Virus titres were measured by $\operatorname{TCID}_{50}$ (C) E gene qPCR (A, B). Statistics for competition assays were determined by One-Way ANOVA with multiple comparisons against time 0 . Statistics for the head-to-head growth curve (C) were determined by multiple unpaired T-tests on log-transformed data. All infections were performed on triplicate wells from matched donors $(\mathrm{N}=3) .{ }^{*} 0.05 \geq P>0.01 ;{ }^{* *} P \leq 0.01$.

Figure 5. Mink and ferret associated mutations have a minimal impact on SARS-CoV-2 antigenicity. reverse genetics-derived WT (D614G) and D614G + Y453F-containing SARS-CoV-2 isolates (B) using $\mathrm{N}=6$ human convalescent antisera from the first UK wave ( April-June 2020; A) or N=10 double-dose BNT162b2 (Pfizer-BioNTech mRNA vaccine) human antisera (B). Fold differences annotated on graph indicate differences in geometric means of $\mathrm{NT}_{50}$. Statistics were determined by two-tailed Wilcoxon test with matched pairs. ${ }^{*} 0.05 \geq P$ 
on graph indicate differences in geometric means of $\mathrm{NT}_{50}$. Statistics determined by matched pair Friedman non-parametric test with multiple comparisons against WT. ${ }^{*} 0.05 \geq P$.

Figure 6. Several variants of concern show enhanced entry into ferret ACE2 expressing cells.

A) Mutant SARS-CoV-2 spike-containing pseudovirus entry into HEK 293Ts expressing human or ferret ACE2, or empty vector. Entry normalised to signals from human ACE2 expressing cells. Each data point indicates data from a completely independent repeat $(N \geq 3)$. Statistics were determined by comparing log-transformed values of ferret ACE2 entry using a one-way ANOVA with multiple comparisons against the WT. $\quad{ }^{*} 0.05 \geq P>0.01 ; \quad{ }^{* *} 0.01 \geq P>0.001 ; \quad * * * 0.001 \geq P>0.0001$;

$* * * * P \leq 0.0001$. RBD mutational profile of the different spike proteins is shown below. Cells in orange indicate changes from WT/D614G. Alpha also known as B.1.1.7; Beta also known as B.1.351; Gamma also known as P.1; Eta also known as B.1.525; lota also known as B.1.526+E484K.

\section{Extended data Figure legends}

Extended data Figure 1. Amino acid differences between ferret and mink ACE2. structure PDB: $7 \mathrm{~A} 94^{60}$.

Extended data Figure 2. Extended data from Figure 3

A) Non-normalised data from Figure 3B. protein and BHK-21 cells expressing the named ACE2 and rLUC-GFP 8-11. Luminescence values shown normalised to human ACE2 (C) or as raw values (D). 
Figures

A
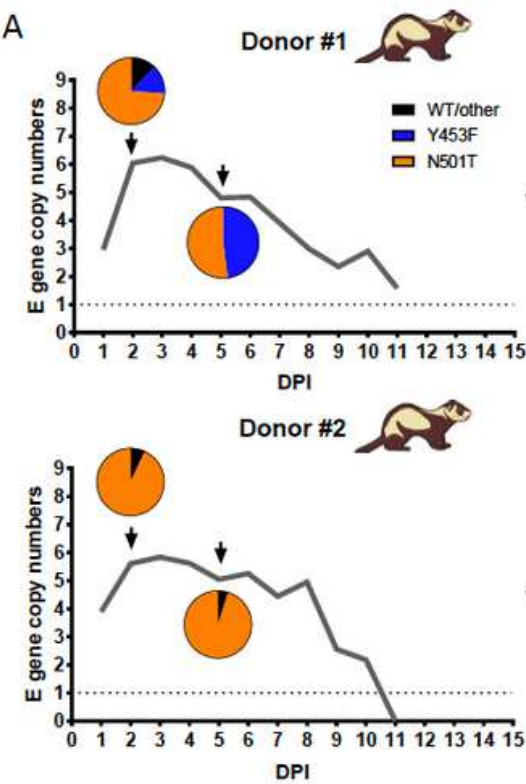
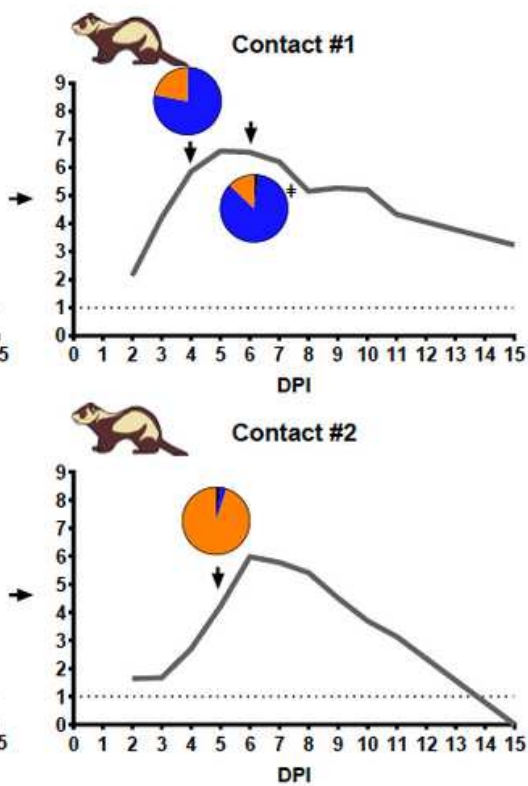

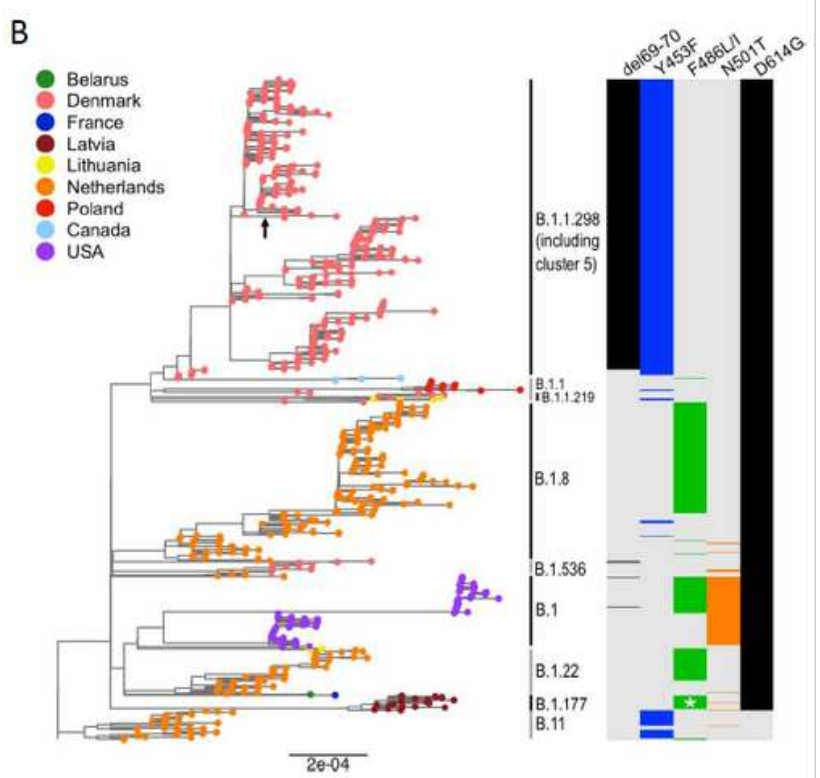

Figure 1

Passage of SARS-CoV-2 in ferrets results in spontaneous emergence of the mink-associated mutations Y453F and N501T. 
A

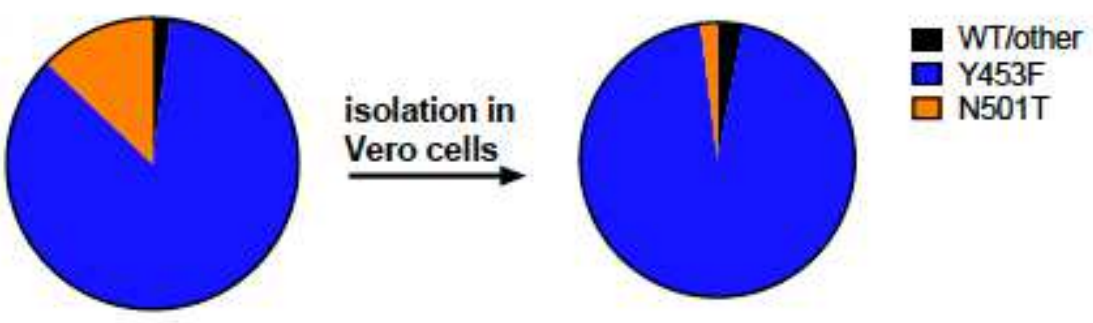

B

C
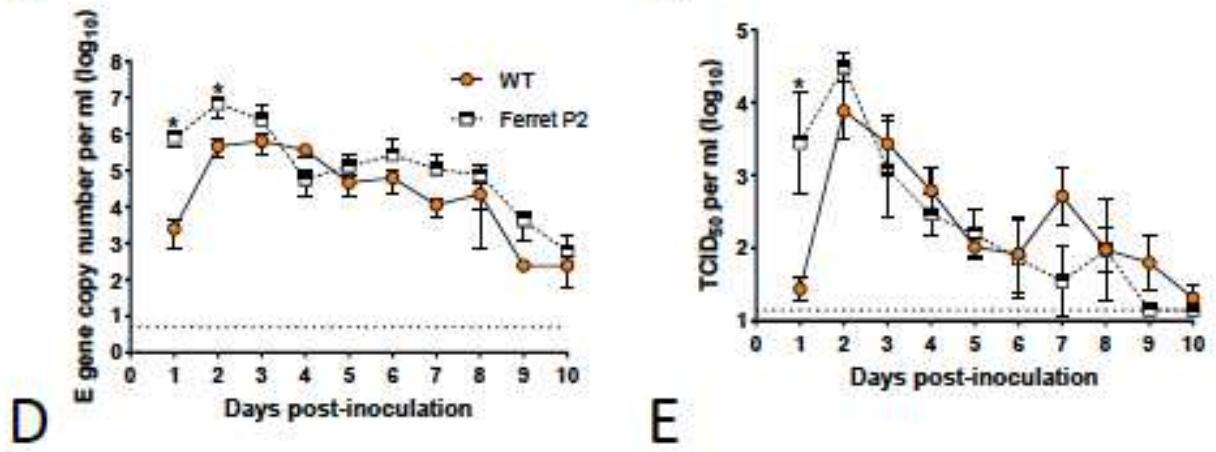

E
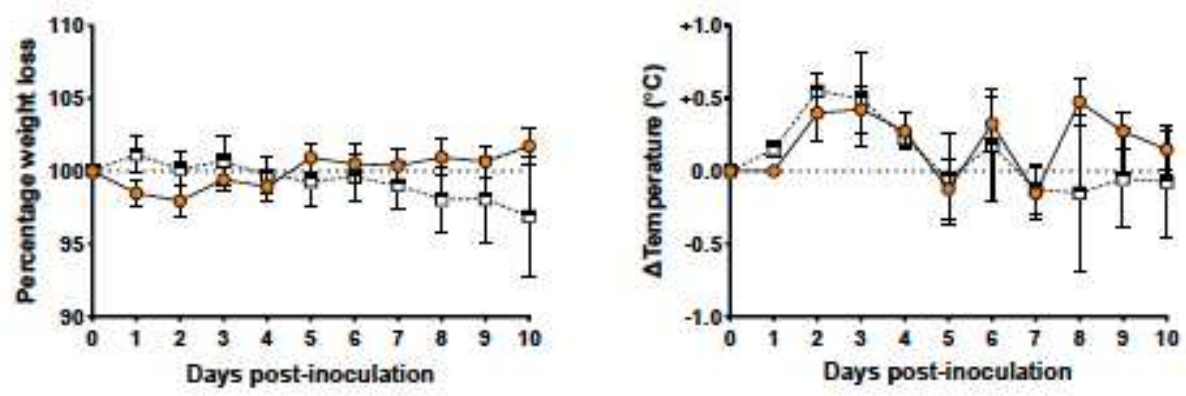

$\mathrm{F}$

G
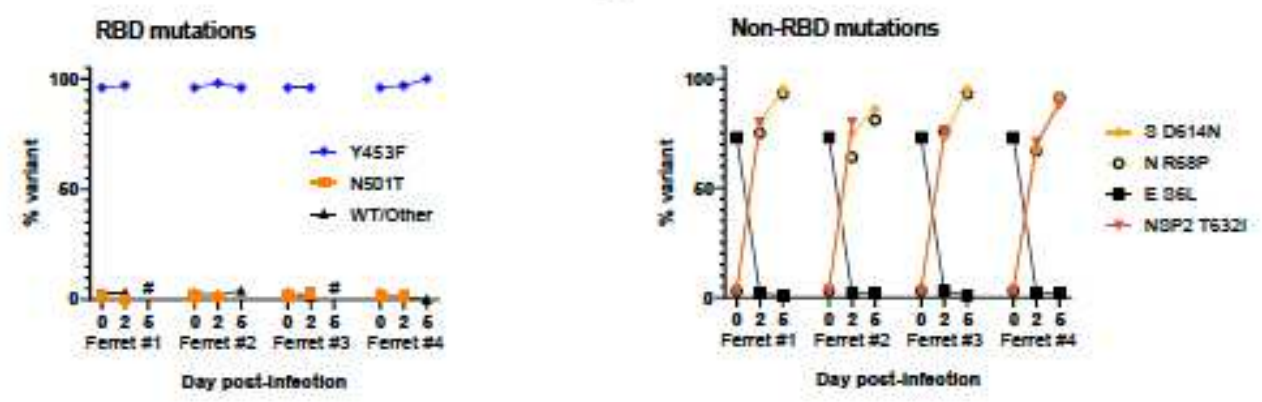

Figure 2

The spike mutation, Y453F, enhances replication and morbidity in ferrets. 
A

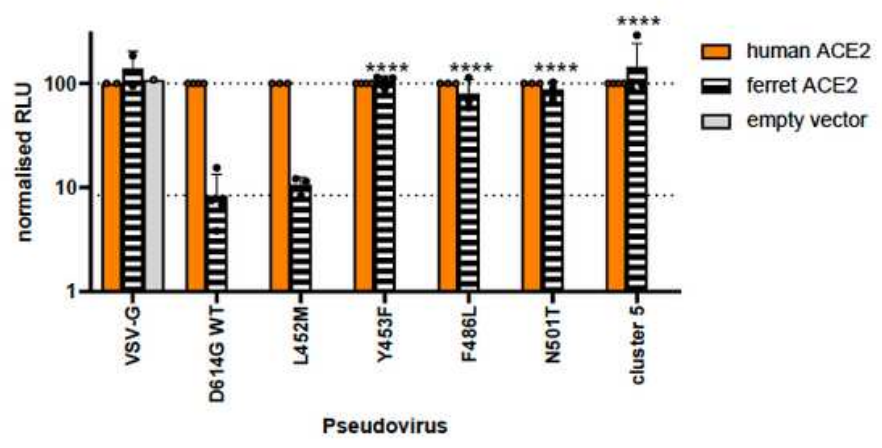

B

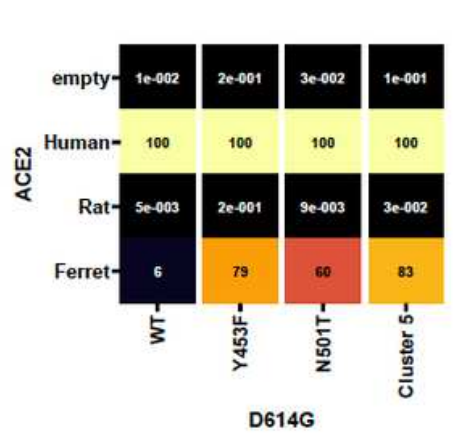

C

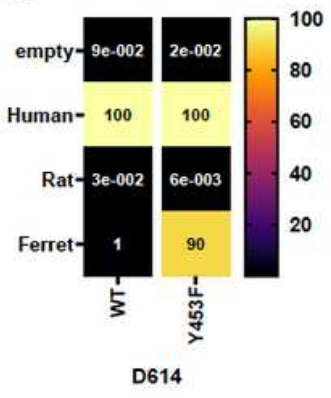

D

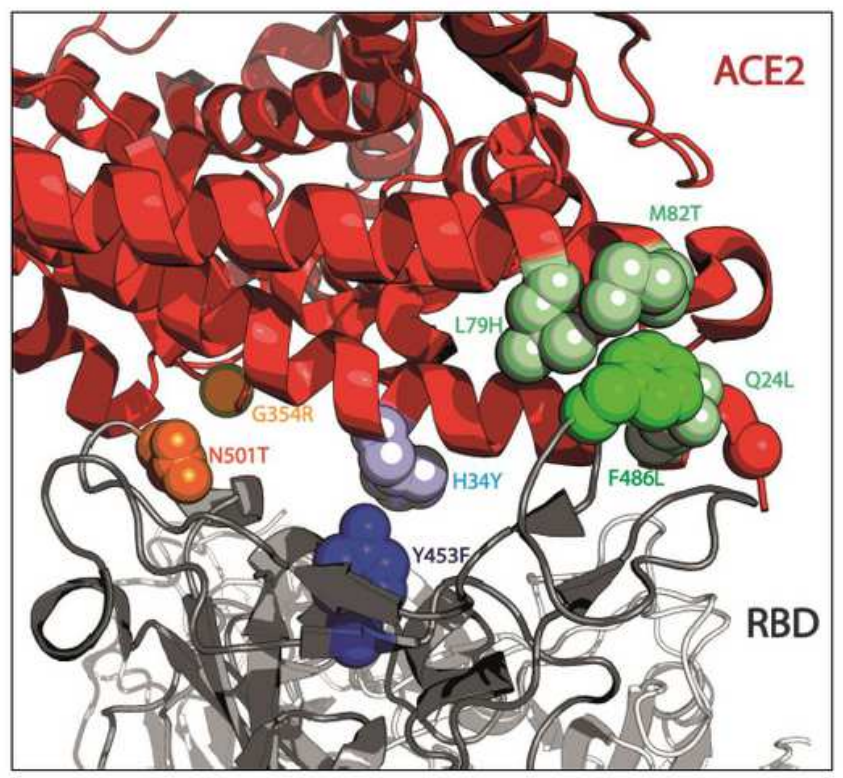

\section{Figure 3}

Mink- and ferret-associated spike mutations allow more efficient entry into cells expressing the ferret ACE2 receptor.

A

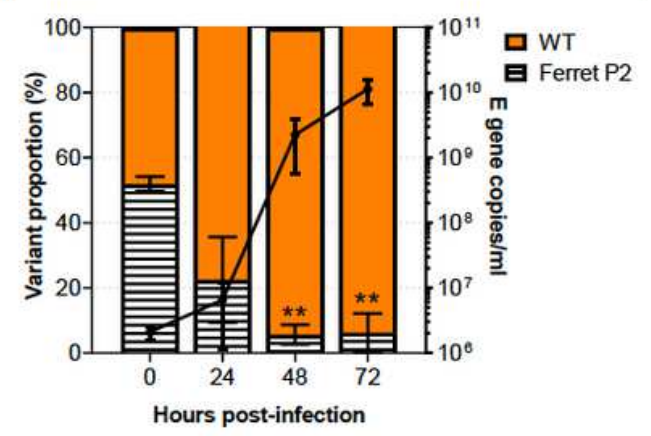

B

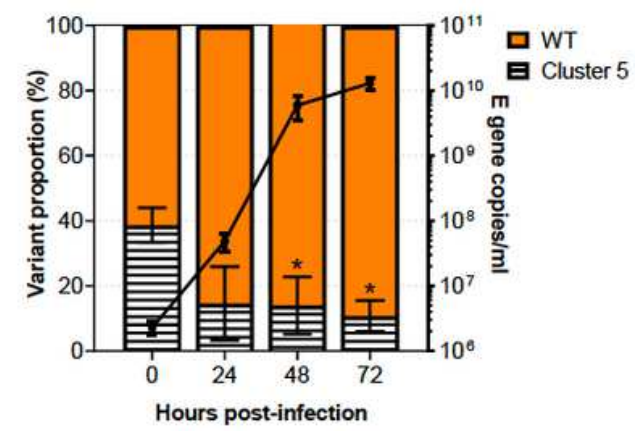

C

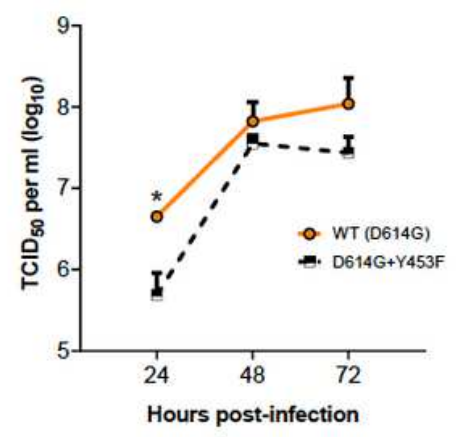

Figure 4

The common mink and ferret adaptation, Y453F, attenuates virus replication in primary human airway cells. 


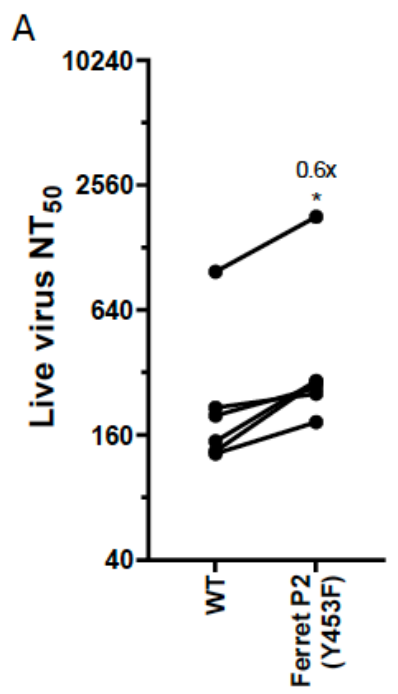

Live virus

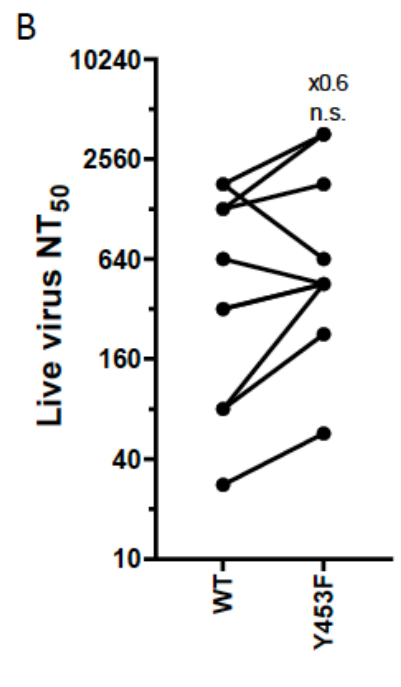

Live RG virus

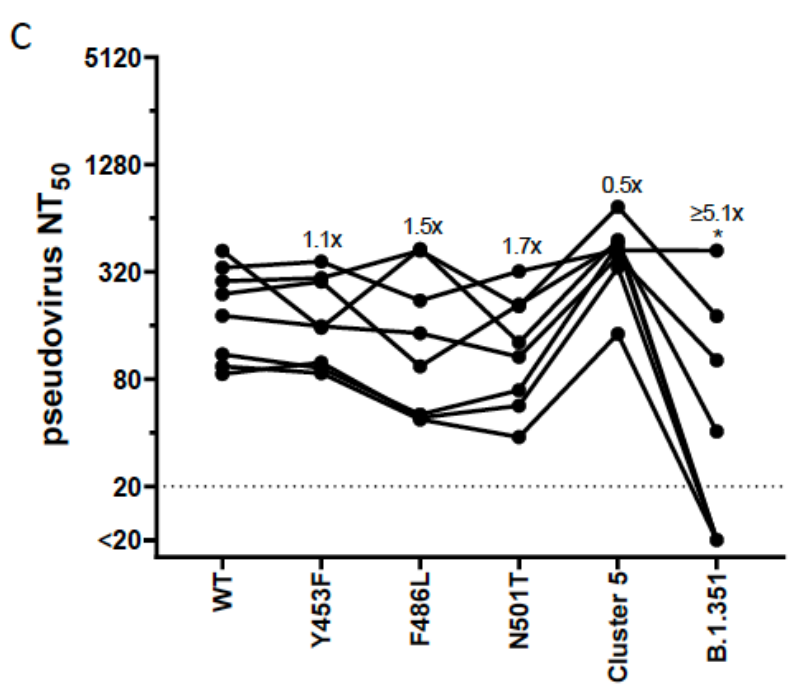

Pseudovirus

Figure 5

Mink and ferret associated mutations have a minimal impact on SARS CoV 2 antigenicity

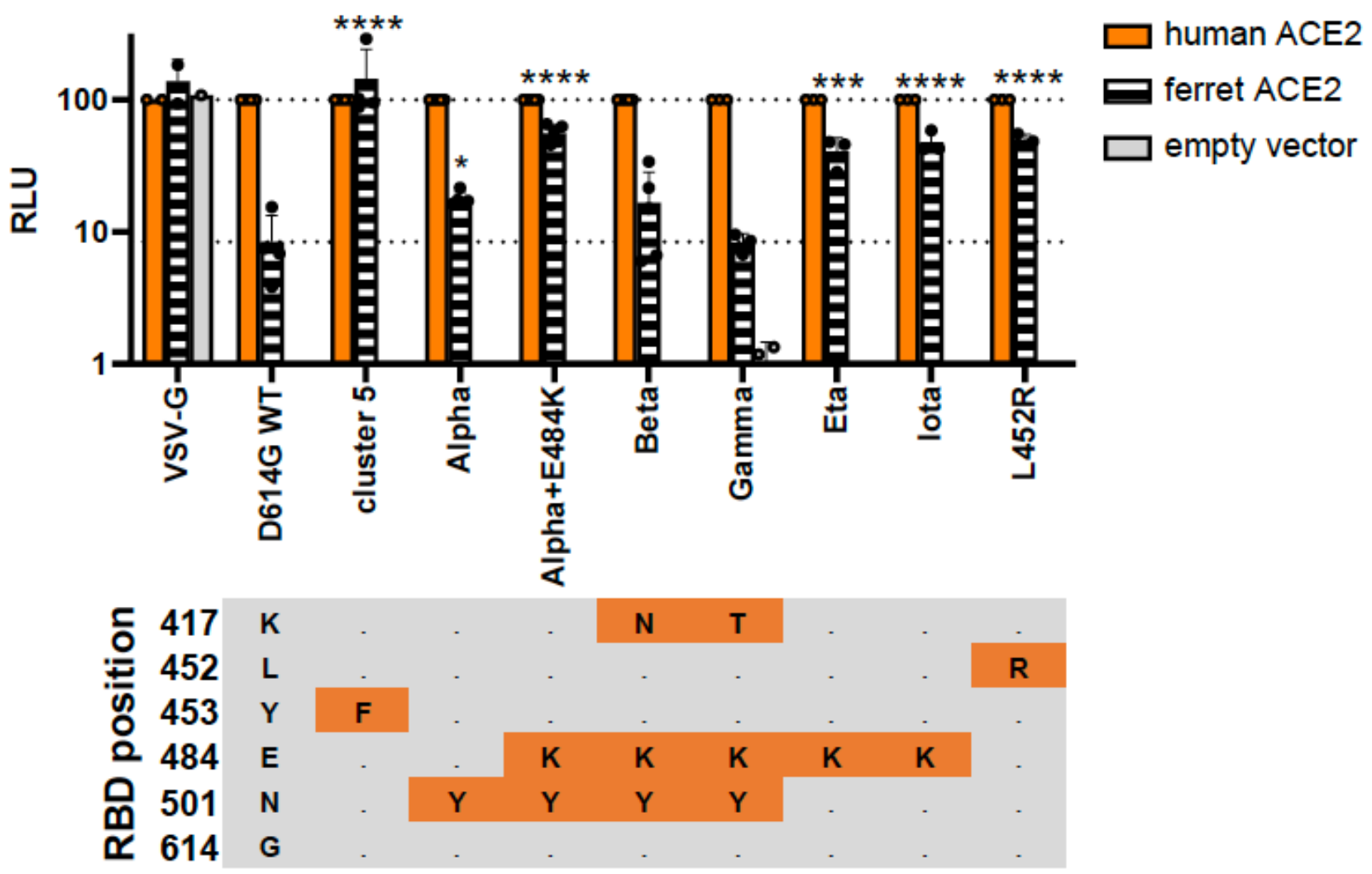

Figure 6 
Several variants of concern show enhanced entry into ferret ACE2 expressing cells

\section{Supplementary Files}

This is a list of supplementary files associated with this preprint. Click to download.

- Slfigures.pdf

- SupplementaryTables1.pdf 